EDITORIAL

\title{
CLINICAL EVALUATION OF NEW SEDATIVE DRUGS
}

THE PRIMARY AIM in the clinical assessment of the efficacy of new drugs is to provide the physician with guidelines in prescribing rationally for his patients. ${ }^{1}$ Several principles have been formulated as a framework for the evaluation of therapeutic compounds. These include the following:

1. The protection of the public by determining unquestionable safety of the drug, first in animals, then in man. Every drug should also meet uniform standards for identity, strength and purity, and each developer should be required to prove that the standards are met.

2. The enforcement of regulatory controls to determine reliably the effectiveness of the drug within the safe dose range by such methods as the double-blind test (including another drug of known action and a placebo (if feasible)).

3 . The making of valid decisions regarding primary uses of the drug based on flexible reliance on the judgment of a panel of non-governmental clinical investigators who have demonstrated expertise in the field of drug evaluation and in the specialties in which the drug may have clinical application.

4. The restraining of commercial advertising until after the safety and efficacy of a drug has been established and the appropriate data published in reputable medical journals.

5. The establishment of post-marketing surveillance to determine whether the new drug is indeed superior to other drugs available, and to keep records of the incidence of serious undesirable effects which might indicate the need of general withdrawal for further controlled investigation.

Gilder noted recently that despite the fact that most French physicians still frown upon the double-blind clinical trial, and do not like to give patients placebos, new draft laws in France call for such government-regulated trials as are done in America, Britain and Canada to establish the effectiveness of new drugs when the latter cannot otherwise be evaluated objectively. ${ }^{2}$

In spite of the rules being laid down in many countries for the testing of drugs, we still do not have good methods to evaluate a drug accurately as to its psychic, pharmacological and physiological effects. This is particularly true when one tries to provide a universal standard for such a common symptom as anxiety. Randomization of treatments, double-blind procedures with placebos and comparable potent drugs, and detailed numerical analyses do not always help in defining our target, which is evaluation of relief from anxiety, with safety and proven efficacy. Even more perplexing is how to decide when a statistically significant difference is clinically significant. Most tests do not indicate whether an observed difference itself is significant, and do not authenticate, validate or annotate the meaning of the difference. Statistical significance can be achieved by a trivial difference if it occurs consistently, or by a meaningless difference if the sample size is large enough. 
Conversely, if the sample size is too small, a difference of major importance will not be "statistically significant."

The inherent problems of using a double-blind technique have also been examined in detail. In order to determine the effectiveness of new sedative drugs, we deliberately try to exclude the therapeutic action of the clinician. This exclusion can provide important scientific data about the value of the drug when used in this isolated manner. However, such an exclusion is morally and ethically wrong in most facets of the practice of medicine. ${ }^{3-5}$

We are acutely aware of these considerations in the practice of anaesthesia because in most instants our patients are very anxious and we have only one opportunity to allay their fears with preanaesthetic drug medication, or by establishing unequivocal confidence in the anaesthetist's care and attention. We hope that the surgeon has done the same.

The primary purpose of preanaesthetic medication is to permit smooth induction of anaesthesia. To accomplish this, it is usually necessary to reduce gastrointestinal and airway secretion, induce amnesia and drowsiness, promote equanimity or tranquility in the face of noxious mental stimuli, and to cause sensory deprivation sufficient to ensure that the patient will not experience pain or psychic disturbances up to the time that a surgical plane of anaesthesia is reached. This goal is attained in as many as 70 per cent of patients, if the anaesthetist merely sees the patient the day before surgery, carefully and compassionately assesses the psychic state, establishes rapport with the patient and then, in the operating room, avoids any procedures that may cause fright, unpleasant discomfort or pain. Management of the environment in a quiet manner greatly influences this self-imposed psychic sedation.

The use of scopolamine usually helps to produce the same desirable psychic state in the patient that can often be induced by the anaesthetist himself. In such a situation it is difficult to determine whether the use of an adjunctive sedative drug substantially adds anything in preparing a tranquil patient. This observation undoubtedly accounts in large measure for the favourable response that appears to occur with placebo medication, and the only marginal advantages apparent with adding active sedative drugs. This occurred in the report of the comparative effect of doxepin, diazepam, secobarbital and a placebo appearing in this issue of the journal. Thus, even though the anaesthetist was not privy to the medication the patient received (excepting scopolamine), there was usually less than a 15 per cent over-all difference in the estimation of adequacy of premedication among the four groups. The categories in the questionnaire in which there appeared to be an advantage with drug therapy was marginal at best, and the anaesthetist frequently could not estimate the feelings and the psychic condition expressed by the patient.

These are some of the basic reasons why many anaesthetists now avoid using sedative drugs, unless there are exceptional indications for their use.

Allen B. Dobkin, M.D.

\section{REFERENCES}

1. Levine, J.; Schiele, B.C.; \& Bouthilet, L. (editors). Prineiples and Problems in Establishing the Efficacy of Psychotropic Agents (Pus Publication No. 21389). United States Government Printing Ofice, Washington, D.C. (1971). 
2. GiLden, S.S.B. London Letter, C.M.A.J., I04: 974 (1971).

3. Feinstein, A.R. Clinical Biostatistics. IX. How Do We Measure "Safety and Efficacy"? Clin. Pharmacol. \& Therap., 12: 544 (1971).

4. Dobknn, A.B.; Wyant, G.M.; \& DYCK, F. Clinical Evaluation of Methyprylon (Noludaro) as a Preanaesthetic Sedative Hypnotic. Canad. Anaesth. Soc. $]_{1}, 4: 27$ (1957).

5. Donkin, A.B. \& Purkin, N. Double Blind Study of Phenothiazines Used in Preanaesthetic Medication. Canad. Anaesth. Soc. J., 7: 158 (1960). 